\title{
Research on Architecture Analysis Modeling and Evaluation means in Aviation Ammunition Support System-of- Systems
}

\author{
SHAO Yunhai, REN Fengyun, ZHANG Li \\ Department of Aviation Ammunition, Air Force Logistics college, Jiangsu Xuzhou 221000
}

Keywords: aviation ammunition; support system-of-systems; complex network; architecture analysis; evaluation means

\begin{abstract}
This paper introduces some modeling means of weapon equipment support system-of-systems first, analyzes the feasibility of aviation ammunition support system-of-systems(AASSoS)based on Complex Networks methods. Then, a network model of the AASSoS architecture was established after introducing the statistics parameter of the Complex Networks and giving the hypothesizes and generating algorithms of AASSoS. At last, by giving the means of evaluation, it shows that the models and the method were effective and scientific in the reality examples.
\end{abstract}

\section{Introduction}

Aviation ammunition support system-of-systems(AASSoS) is defined as the higher level system. With the certain structure and comprehensive integration, this system is made up of the interrelated and superiority complementary aviation ammunition support force, support resources, functions and links during the process of aviation ammunition support works. In a sense, Each aviation ammunition support entity is the basis of AASSoS when it operates. Architecture determines the function of the system, a rational and scientific architecture plays an important role during the whole system operating. Using modeling methods to get the various nature of the architecture of AASSoS is the origin that we attempt to understand the character of aviation ammunition support. This paper based on complex network theory, by building a network model of AASSoS, correspond the complex network of statistical features to AASSoS structural performance indicators, analysis and evaluate the overall performance of AASSoS. The conclusion in the paper is benefit for structuring the future AASSoS which adapts information warfare and supply a certain reference.

\section{Modeling of Equipment Support System overview}

Equipment support system modeling, mainly for describing in the objective explanation, which contains system function, constituent elements, information transfer mode, operation mechanism etc, which is the basis for quantitative research. Therefore, choose the appropriate modeling method has a vital role for researching influence of hierarchy and operation mechanism in AASSoS to make the best AASSoS building programs.

\section{Common support system modeling.}

In the field of research equipment support system, from the retrieve data during the current situation, most of the researches are based on those five aspects of system modeling: functions, organization, resources, information, process.

(1) System Function Modeling

The main purpose of functional modeling is to describe the running function of the system. In order to realize the ultimate goal of the system, what work or task the system should be completed. System function modeling not only contribute to the management and control systems, but also provides for improved target that in functional optimizing. Currently, there are MFM (Multievel Flow Modeling) method, GTST (Goal-Tree Success-Tree) method, and IDEF0 (ICAM Definition Method) method.

(2) Support Process modeling

By means of defining the component activities and the logical relationship between activities, 
support process modeling can describe the designing process of the support, It uses a computer-understanding and dealing language, an accurate description of the process of running equipment support for process analysis and design optimization of use. Currently the more common support process modeling methods are IDEF3, Petri nets, State diagrams, behavior-based language theory, ARIS analysis means etc.

(3) Support Information Modeling

Support Information Modeling reacts the flow of support activities information profiles, and abstracts the support, technology, processes and management information of internal entity. By using support information model, describing the exchange of relevant information, we can achieve the connection between the various support entities and systems. Currently, the main methods of support information modeling are data flow diagram (DFD) method, entities associated with Figure (ER) method, IDEF1x law, Yourdon design method, Jackson Design, Perti network, GRAI method, UML methods etc.

(4) Support Resource Modeling

Support resources modeling describes the support system internal resource forms and use of internal resources to implement the system configuration and scheduling, through the description of belonging resources composition, structure, properties, achieve the usage of resource in the system optimizing and efficiency. The common modeling methods are IEM and CIM-OSA etc.

(5) Support Organization Modeling

Organization modeling is a kind of using abstract models and elements to construct a series of relationships in the organization for the expression of the relationship between organizational entities and organizational entities. Organization modeling can describe the relationship between organization tree, personnel, capabilities, roles, and permissions among the cells. They can achieve organizational reconstruction, and improve institutional flexibility and agility of external support, but also promoting the support for management entity approach, analysis and optimization, achieving the optimal allocation of the organization. Currently, common modeling methods are CIM-OSA method, based on theoretical modeling of complex networks etc.

\section{Select modeling method.}

Though there are lots of methods of support system modeling, for AASSoS architecture performance, our purpose is exploring the relationship between support hierarchy and operation mechanism in the whole AASSoS performance, then, according to the conclusion, to build the future AASSoS which adapts information warfare and supply a certain reference. Analyze the five above modeling methods, support organization modeling can reach this goal, especially the complex network method, it is more scientific and effective.

Research on complex network theory can be traced to the famous "Seven Bridges Problem" during the 18th century. Paper [2],[3] found no scaling feature complex networks and small world phenomenon, the complex network research come to a new era. The research is no longer limited to the scope of mathematics, more and more researchers pay close attention to the research of complex networks. Recently, complex networks ideological plays an important role in guiding power transmission systems, transportation systems, and social management systems and many other fields, which have achieved good results. Some researchers are trying to apply the complex network theory to the military field. Using the complex network theory on the AASSoS modeling, its feasibility are mainly in the following two ways:

(1) Similarity in architecture

Firstly, compared with AASSoS and complex networks, they are all the systems, they have the common characteristics of systems. Secondly, they all have lots of entities in the systems and the relationships between the entities are exceedingly complex. Thirdly, there are resource and information flow on the lines, Attributes of the line is influenced by the length and weight of the line. above all, we consider that AASSoS is a kind of complex networks.

(2)Reference from other researchs

Paper[4] , [5] , [6] , [7]from different angles of view researched the equipment support system and $\mathrm{C} 2$ system, the conclusions show that equipment support system have the whole characteristic 
of complex network, AASSoS is the important part of our army equipment support system, these research have supplied a good reference, so we decide to use complex network theory to research the AASSoS.

\section{Characteristic parameters of complex networks}

People come out many concepts and methods in describing the statistical properties of complex network structures. This paper focuses on the following basic concepts, including average path length, clustering coefficient, Average degree and degree distribution.

\section{Average path length.}

Defined path length between any two communicating $d_{i j}$ nodes for all $(i, j)$ path, the path of least through other nodes (shortest path) of the number of lines, and the average path length $\mathrm{D}$ :

$$
D=\frac{2}{N(N-1)} \sum_{i \geq j} d_{i j}
$$

$\mathrm{N}$ is the number of nodes in the network.

Average path length can measure the transmission characteristics and connectivity information between network nodes, usually when $\mathrm{D}$ is relatively small, information, materials or energy exchange faster. For example, in a social communication network, reduce the average path length of the network, namely the reduction of the turnover from the dissemination of information, thus improving the timeliness of information dissemination.

\section{Clustering coefficient.}

Any node $j$ in the network, directly connected nodes called $j$ adjacent node. Clustering coefficient set its adjacent nodes is $E_{j}$, the actual number of lines between these nodes is $C_{k_{j}}^{2}$, the $j$ node clustering coefficient $C_{j}$ is:

$$
C_{j}=\frac{E_{j}}{C_{k_{j}}^{2}}=\frac{2 E_{j}}{k_{j}\left(k_{j}-1\right)}
$$

Namely, you can also get all the nodes clustering coefficient, which is the average of the average clustering coefficient called $C$.

$$
C=\frac{1}{N} \sum_{i=1}^{N} C_{i}
$$

The average clustering coefficient is designed to measure an important parameter network node clustering degree. In general, the larger the average clustering coefficient indicates links between network nodes more closely, when adding or removing a node, less impact on the entire network, because the average clustering coefficient larger network, two nodes may be more than one path between, after the removal of a node, transmission of information or objects can be achieved through other routes.

\section{Average degree and degree distribution.}

Degree for $i$ is $k_{i}$ that defined as the number of nodes that connected to node $i$, the average degree of the network is that the mean value of the degree. Sign it $\langle k\rangle$, The distribution of the degree of nodes in the network can be described by the distribution function $p(k)$.

The degree of network can be considered as the number of lines connected to the node. The average degree reflects the number of that nodes in the network lines have the average lines. It also reflects the nature of the network, namely, in the same network, the greater the average degree the more number of lines there are in the network, Further more that reflect the complexity of the network. 


\section{Build AASSoS network modeling}

The hypothesis of the modeling.

(1)Nodes. According to the function the nodes of AASSoS can represent professional management departments, ammunition warehouses, repair depot, execute teams etc, in order to be consolidated and universal, we regard all the entities as a node.

(2)Lines. The lines represent the relationship among all nodes, not only professional work, but also geographical position. we mainly build the AASSoS networks focus on professional work, so we set AASSoS connecting nets.

(3)The ability of all the type of the nodes is the same. they have the same directions of information, energy. So this net is a kind of 0-1 unweighted network.

The generate algorithm.

Step_1:Set the number of management nodes Num_GL, set the span in every level, the number of the span is $M 1, M 2, M 3 \cdots \cdots$;

Step_2: Generate the center nodes, that is the first node, set the level number is $L=1$;

Step_3:Accroding to the span $M$, Generate the next level nodes and connect each other;

Step_4: Repeat Step_3, until the management nodes reached Num_GL,management nodes networks finished;

Step_5: Generate Num_CC storage nodes and Num_XL repaired nodes.

\section{The means of AASSoS performance evaluation}

\section{Performance Indicators.}

(1) Timeliness index

Time-efficient index reflects the speed of logistics, information flow in AASSoS. The shorter distance between nodes, the faster transmission of support information, Combining equation 3.1, we assume timeliness index of AASSoS expressed by $S$, so there is $S \propto \frac{1}{D}$, whereas the existence of timeliness index factor, here we definition:

$$
S=\frac{1}{\mu D+1}
$$

$\mu$ is the timeliness index factor of AASSoS.

(2)Robustness index

Robustness is an important indicator of system performance, it is the ability maintaining its normal function when the system affected by external changes, Combining equation 3.2, we assume the robustness of indicators of AASSoS expressed by $R$, so there is $R \propto C$, whereas the existence of robustness index factor,here we definition:

$R=e^{-1 / \theta C}$

$\theta$ is the robustness index factor of AASSoS.

(3)Complexity index

The increasing contacts in the network will bring some predict harmful effects: First, increasing the construction cost of the network. Second, making the management level fuzzy, management control emerged complexity. The most direct reason of the complexity in the network is the large quantity of the links among nodes. in complex network modeling, the average degree react the connection of nodes, combining equation 3.3, we assume the complexity index of AASSoS expressed by $H$, so there is $H \propto K$, whereas the existence of complexity index factor, here we definition:

$$
H=1-e^{-\varepsilon K}
$$

$\varepsilon$ is the complexity index factor of AASSoS. 
Those three indicators reflect different aspects of the overall performance of the AASSoS. Its correspondence with the complex network characteristic parameters are shown in Figure 5-1.

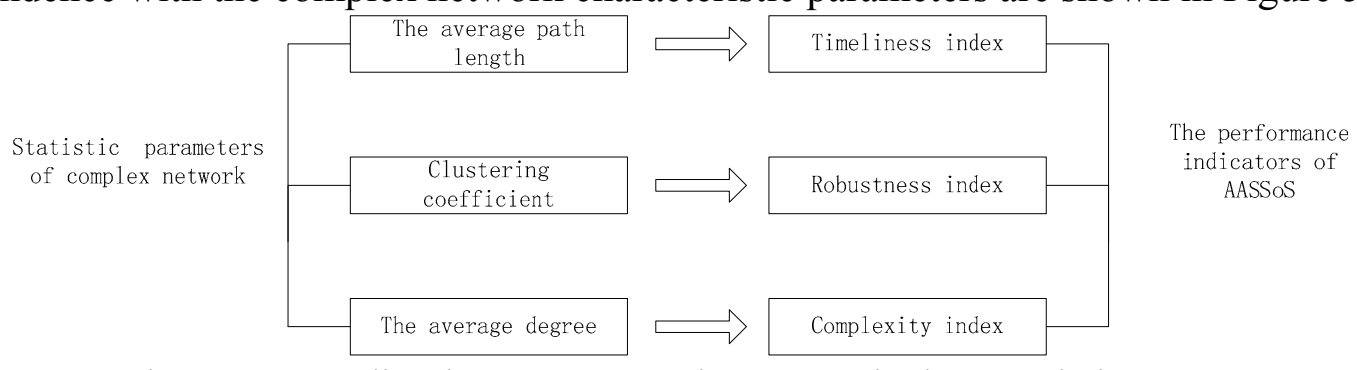

Figure 5-1 The corresponding between Complex network characteristic parameters and the performance indicators of AASSoS

\section{Estimate function.}

We consider that the performance of AASSoS expressed by $U$ is made of timeliness index, robustness index and complexity index.

$$
U=f(S, R, H)
$$

Among those three indexes, timeliness index and robustness index are beneficial for the whole system, but, complexity index is pernicious for the system. So we define:

$$
U=\omega_{1} \frac{1}{\mu D+1}+\omega_{2} e^{-1 / \theta C}+\omega_{3}\left(1-e^{-\varepsilon K}\right)
$$

$\omega_{1}, \omega_{2}, \omega_{3}$ are the weight factor, they are different, because there are lots of different significance in timeliness index, robustness index and Complexity index among the different system. We compute $\omega_{1}, \omega_{2}, \omega_{3}$ by G1 means in systems engineering, and set $\mu=0.2, \theta=4, \varepsilon=0.8$. So estimate function of AASSoS:

$$
U=0.45 \frac{1}{0.2 D+1}+0.30 e^{-1 / 4 C}+0.25\left(1-e^{-0.8 K}\right)
$$

\section{Applications.}

Whereas the confidentiality requirements, we process the relevant data proper, according to the proposed method in this paper, We use UCINET software to make the support system a visual sight, Figure 5-2 shows the AASSoS network. Learn from the successful experience of the US military logistics construction, We assume: (1) Integrate some departments of AASSoS to optimize the support functions. (2) Under the same affiliation, establish the horizontal relations among the grassroots support team.(3) establish the striding relationship between campaign and tactical levels. The optimized AASSoS network topology shown in Figure 5-3:
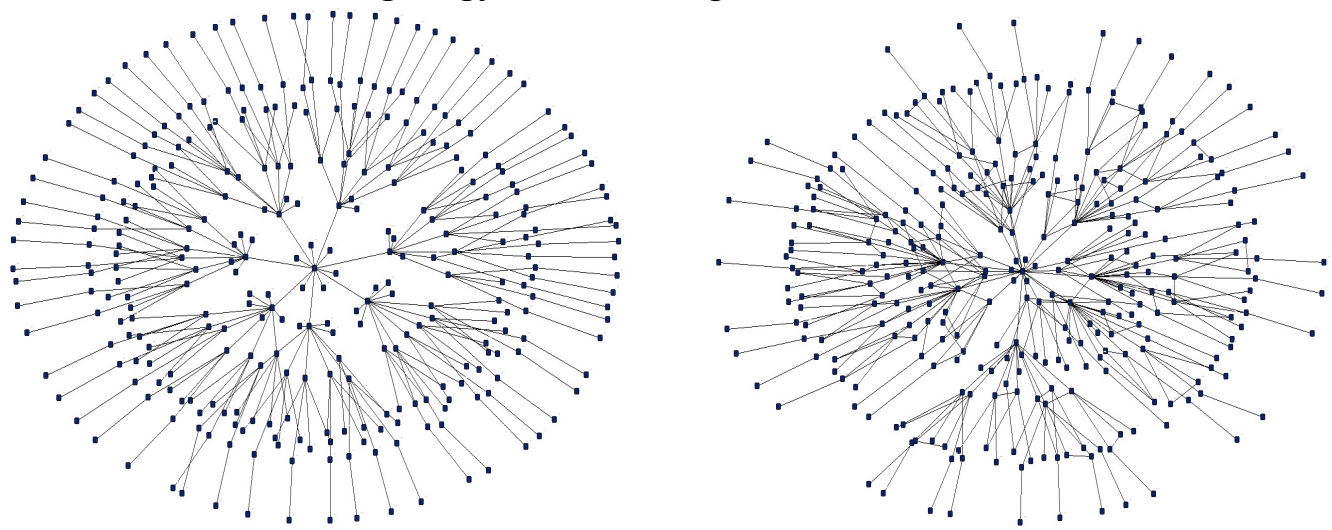

Figure 5-2 AASSoS network topology

Figure 5-3 optimized AASSoS network topology

Using matlab7.1 software calculated each performance index value and the overall performance function value of AASSoS. That is shown in Table 5-1. 
Table 5-1 AASSoS network characteristic parameter table

\begin{tabular}{ccccc}
\hline Name & Aging index & $\begin{array}{c}\text { Robustness } \\
\text { Index }\end{array}$ & $\begin{array}{c}\text { Complexity } \\
\text { Index }\end{array}$ & $\begin{array}{c}\text { Performance } \\
\text { function value }\end{array}$ \\
\hline $\begin{array}{c}\text { Original } \\
\text { system }\end{array}$ & 0.2658 & 0.0000 & 0.7523 & 0.0054 \\
\hline $\begin{array}{c}\text { Optimized } \\
\text { system }\end{array}$ & 0.5093 & 0.2707 & 0.8681 & 0.1622 \\
\hline
\end{tabular}

As can be seen from Table 5-1, optimized system is better than the original system, mainly because of the focused tasks of the support entities, and the flat degree of the network.(implementation of integrated support team shares, reducing the number of nodes in the network), At the same time, support is also inseparable from the degree of interoperability between entities guarantees a higher level of organic combination (establish the cross-level command and peer support mechanisms). However, such improvements brought the increasing complexity of AASSoS, bring the negative influence to the AASSoS. This tells us that when we optimize our AASSoS in the near future, we must demonstrate the relationship among science, timeliness and complexity, on the basis of efficient, we should think about that the entire system can not be too complicated, try to find "the balance" to avoid "overkill" to maximize the performance of AASSoS.

\section{Conclusion}

This paper introduces the basic methods of equipment support system modeling, elaborates the feasibility of researching the AASSoS by using complex networks, lists the basic characteristic parameters of complex networks, and gives the generation algorithm, and establishes a network modeling of AASSoS. Finally, by giving the performance index and evaluation function of AASSoS, the paper calculates each performance index value and the overall performance function value. By comparing the optimized system and original system, the paper gives the scientific judgment.

\section{References}

[1] Wang Xiaofan. Li Xiang. Chen Guanrong. Complex network theory and its application [M]. Tsinghua University Press. 2006.04

[2] Watts DJ, Strogata S H.Collective dynamics of 'small-world'networks.Nature, 1998,393 (6684); $440 \sim 442$

[3] Barabasi AL, Albert R.Emergence of scaling in random networks.Science, 1999,286 (5439)

[4] Zhang Yong, Yang Hongwei, Bai Yong. Empirical Research on Equipment Support Network complex network theory [J]. Equipment Institute. 2014 (01): 83-86

[5] Han Zhen. LU Yu. Gu Ping. CHEN Li-yun. Maintain Support System modeling of complex network-based approach [J]. Fire command and control. 2014 (09): 31 to 35

[6] Liu Yadong, Zhang Chunrun, Linghu Changying. Construction Based Equipment Support Resources System complex networks. Ordnance Engineering College [J]. 2013 (02): 1-6

[7] Liu Yue. Based on the allegations tissue modeling and assessment of complex networks [D]. Changsha: National University of Defense Technology, 2012,11 